\title{
Unlocking Perceived Algorithmic Autonomy-Support: Scale Development and Validation
}

\author{
Nura Jabagi ${ }^{1}$ \\ JMSB \\ Concordia University \\ $\mathrm{n} \_$jaba \\ @jmsb.concordia.ca
}

\author{
Anne-Marie Croteau \\ JMSB \\ Concordia University \\ anne-marie.croteau \\ @ concordia.ca
}

\author{
Luc K. Audebrand \\ FSA \\ Université Laval \\ luc.audebrand \\ @ fsa.ulaval.ca
}

\author{
Josianne Marsan \\ $\mathrm{SIO}$ \\ Université Laval \\ josianne.marsan \\ @ sio.ulaval.ca
}

\begin{abstract}
Platform workers' autonomy and agency are recurring themes in the study of the gig-economy where narratives purporting workers' autonomy and empowerment conflict with those alleging the control and marginalization of workers. While it has been said that promoting workers' agency can threaten the valuation of platform-based companies, the benefits of supporting workers' autonomy in traditional organizations are well-established. To understand such inconsistencies, it is necessary to measure perceptions of autonomysupport; yet, no validated instruments exist that can be used to measure workers' perceptions of algorithmic autonomy-support. To address this gap, we draw on the Theory of Self-Determination to reconceptualize the notion of autonomy-support for the technoorganizational phenomenon of algorithmically managed platform work. In doing so, we introduce a new construct, namely: Perceived Algorithmic Autonomy Support (PAAS). In this work-in-progress paper, we describe our current work in developing and validating a theoretically-based measure for PAAS. Preliminary results are provided.
\end{abstract}

\section{Introduction}

In 1950, Alan Turing, the progenitor of modern computing, wrote: "The idea behind digital computers may be explained by saying that these machines are intended to carry out any operations which could be done by a human computer" [1]. Though artificial intelligence was in its embryonic stages at the time, Turing, along with other computer scientists such as Licklider, already viewed the roles of humans and machines as intertwined [ibid.]. Now, 70 years later, organizations are still exploring ways to unlock the full potential of technology, to free humans of repetitive tasks, and to enable better decision-making.

In this pursuit, the roles played by information technologies and humans have become even further enmeshed in recent years with the birth of the gigeconomy. Fuelled by a new era of Big Data collection and advancements in machine learning techniques, algorithms have gained the ability to continuously learn and adapt as they make decisions and are exposed to new data. This ability has not only empowered algorithms to provide advanced decision support to humans, it has enabled them to assume responsibility over managerial practices, performing complex tasks typically done by middle and upper management $[2,3]$.

The application of algorithms to the management of workers on digital labour platforms is a sociotechnical phenomenon known as algorithmic management, or management-by-platform in the gig-economy [4]. Whilst it is tempting to consider algorithmic management as akin to the use of decision-support systems by managers, this set of managerial practices constitutes more than just a repackaging of pre-existing technical competencies and resources in organizations. Rather, algorithmic management signifies a unique, and entirely new, managerial logic where the minimization of human intervention is requisite for organizations to benefit from algorithmic-efficiencies [2, 3, 5].

Yet where programming language is the key organizational structure, and when human managers are replaced by source code, once overt organizational and managerial processes are "reduced into a set of opaque algorithmic processes that are both complex and inaccessible to the typical worker" $[3$, p. 3]. Thus, the outcome of algorithmic management is a lack of transparency in these sociotechnical ecosystems, a situation which labour platform owners have leveraged to narrow workers' decision-making capacities and to reduce their autonomy while sustaining the veneer of entrepreneurial labour [2, 5, 6, 7, 8, 9].

Where the promise to "be your own boss" is a key recruitment tactic in the gig-economy, and the desire for autonomy has been cited by gig-workers as a leading driver for participation [10], the effects of algorithmic management on workers' perceived autonomy can have significant impacts on workers' participation in the gig- 
economy, their attitudes and experiences, as well as organizational performance $[2,3,5,6,7,11]$. Notably, in traditional work environments, numerous studies have demonstrated that autonomy-supportive work contexts lead to increased motivation, performance, and job satisfaction, as well as improved psychological and physical well-being and reduced absenteeism $[12,13]$.

Given evidence that managers play a key role in supporting workers' autonomy [12], it is of theoretical and practical importance to understand how perceptions of autonomy-support are formed when workers are managed by faceless technology, and interaction is increasingly, if not entirely, mediated by computing devices. Notably, IS scholars such as Orlikowski and Scott [14] have highlighted the need to explore the sociotechnical aspects of algorithms on managerial practices [15]. While research into users' perceptions of algorithms is starting to grow, currently, there seems to be no instruments that can be used to measure platform workers' perceptions of algorithmic autonomy-support. Moreover, the gig-economy literature is rife with conflicting accounts of autonomy and empowerment versus exploitation and marginalization $[4,10]$.

The goal of this paper is to develop an instrument that addresses the absence of reliable measures available to conduct research on gig-workers' experienced autonomy. To do so, we draw on Self-Determination Theory (SDT) to reconceptualize the notion of autonomy-support for the context of platform-mediated work. Guided by established scale development procedures [16, 17, 18], we detail the steps taken to develop and validate a measure for a newly proposed construct: perceived algorithmic-autonomy support (PAAS). Our systematic approach includes an extensive search of the literature, interviews with Uber drivers, as well as a sorting exercise with academic experts and Uber drivers. With a preliminary instrument developed (33 items) and validated for content adequacy, our next step is to examine the scale's psychometric properties and to validate the scale within its nomological net. A large-scale survey study is currently underway.

Our paper is structured accordingly: We first review the theoretical background of autonomy-support in work contexts. Next, we discuss the notion of autonomy in the context of the algorithmically managed gigeconomy. In doing so, we introduce our new construct, PAAS, and the challenges in measuring PAAS. This is followed by our instrument development process and a discussion of our in-progress survey study. We conclude with our expected contributions and next steps.

\section{Theoretical Background}

The notion of 'autonomy-support' derives from selfdetermination theory (SDT). The need for autonomy refers to an individual's inherent desire to experience a sense of choice, volition, and psychological freedom when engaging in an activity [12]. Importantly, being autonomous does not mean being independent, nor does it entail being free from responsibilities. Rather, autonomy is experienced when one acts in a way that is aligned with their self-endorsed values and interests [12, 19]. Thus, an autonomy-supportive work context refers to an environment where managers consider workers as being distinct individuals capable of self-determination, and behave accordingly.

SDT scholars have conceptualized 'autonomysupportive' work contexts as a cluster of managerial behaviours that lead to the satisfaction of one's need for autonomy. Specifically, such contexts are denoted by managers: acknowledging employees' perspectives and asking for their viewpoints; providing rationale when requesting tasks; offering choices with regards to how to do aspects of their job; empowering decision-making and self-initiation; and providing positive and/or meaningful feedback [12]. In view of this cluster of behaviours, autonomy-support is generally considered to be a second-order formative measure [19].

While the notion of autonomy-support has been explored extensively in traditional management contexts - where human managers supervise and support full-time employees, often by building close, trust-based relationships - there is significantly less research exploring the concept in non-traditional contexts where advanced technologies mediate interactions and, very often, form the work environment $[3,12]$. Thus, insofar as gig-workers lack an official human supervisor, but rather are managed by algorithms, it is necessary to reconceptualize the notion of autonomy-support in view of the manual, cognitive, and affective impacts of "algorithmic managers" on the interpersonal context.

\section{Autonomy-Support in the Gig-Economy}

Digital labour platforms have been conceptualized by some as sociotechnical assemblages encompassing both the "technical elements (of software and hardware) and associated organisational processes and standards" $[9$, p. 126]. In accordance with such conceptualizations, and following the work of Sutherland and Jarrahi [20], we further recognize that digital labour platforms can be conceptualized as a set of technological affordances where there is a trade-off between the agency that platform features (e.g., algorithms) take in conducting transactions and the amount left to users.

Applying this conceptualization, digital labour platforms can be classified along a continuum ranging from highly decentralized platforms (which rely on the 
discretion, and thus autonomy, of users to conduct exchanges) to centralized models (which automate and take control of exchanges). Given that algorithmic management was initially developed to optimize the convenience, speed, and seamlessness of undifferentiated, low-skill on-demand service exchanges (e.g., Uber) [5], we focus our attention, to these highly centralized digital labour platforms.

In the context of the gig-economy, a gig-worker's autonomy is "typically defined in terms of their ability to self-schedule when they work, their right to reject or accept gigs and, depending on the platform, their ability to choose the methods and processes they use to conduct their work" [3, p. 4006]. Given that companies that promote workers' autonomy through the right to accept or decline work-orders and the right to self-schedule tend to experience coverage issues and reduced profit margins, gig-organizations are known to counter workers' autonomy through "softer" and less visible forms of control [3, 6]. In addition to platform surveillance, such tactics take hold through intentional information asymmetries that constrain workers' decision-making capacities and making it difficult for workers to gain control over their work, thereby reducing their autonomy. Thus, insofar as platform algorithms are designed to manage and control workers, a gig-workers' autonomy can be conceptualized as a function of a platform's algorithms [2, 6, 15, 20].

Where algorithms implemented in management contexts operate a set of rules (or instructions) that embody an organization's policies and procedures, managerial algorithms not only shape workers' autonomy through job-design, they may also do so by acting as a "social agent" of the organization [3]. According to the Computers Are Social Actors (CASA) paradigm [21], by filling roles traditionally assigned to humans, using words for communicating with the user, and exhibiting high interactivity, managerial algorithms on digital labour platforms provide sufficient basis to cue "humanness" and to encourage social responses via the treatment of computers as social actors [3].
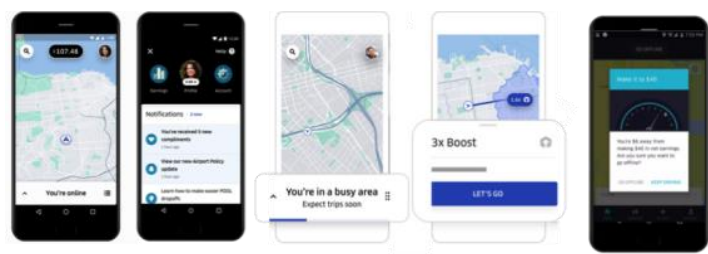

Figure 1: Uber Platform Interface

For example, Figure 1 depicts the Uber platform interface where algorithms replace humans as managers, and where the interface uses words for communicating with the user while also demonstrating high interactivity with the driver based on personalized information gathered via the digital device that connects a worker to the platform.

Building on the proposition that in the absence of human managers, gig-workers will respond to managerial algorithms as though they are social agents of the organization [3], we suggest that the design of such an algorithm will play a key role in generating an 'autonomy-supportive' work context that leads to the satisfaction of a gig-worker's need for autonomy. Notably, this is aligned with recent research proposing that the operational choices embedded within a platform's architecture will implicitly shape platform workers' autonomy such that platforms can be either autonomy supportive or controlling in respect of various managerial behaviours highlighted in the SDT literature $[6,11,15]$. For instance, Jabagi et al. [15] proposed that platforms that allow workers to rate clients and/or offer recourse for unfair ratings/poor treatment by clients can be viewed as "acknowledging workers' perspectives" and thereby embody autonomy-supportive contexts.

Accordingly, we introduce the concept of perceived algorithmic autonomy support (PAAS) which we broadly define as the degree to which a platform worker perceives that an algorithmically-managed digital labour platform is autonomy-supportive.

\section{Challenges in Measuring PAAS}

In reviewing the literature, we found no existing measures of perceived algorithmic autonomy support, nor of autonomy support for algorithmically-managed work contexts. In its application to the workplace, SDT scholars have measured "autonomy-supportive" interpersonal contexts using the Work Climate Questionnaire (WCQ) [12]. The WCQ has both a long form (15 items) and a short form (6 items). A review of the instrument reveals that it cannot be used, as it stands, to assess autonomy-support in algorithmically-managed contexts for various reasons.

Firstly, where gig-workers are managed algorithmically, any references to a human manager in the items would need to be adapted to "algorithm" or "platform". However, in many cases, various items cannot be straightforwardly adapted from referencing a "human manager" to an "algorithm". For instance, adapting Item 11 would yield the following statement: "The algorithm [platform] handles people's emotions very well". Such an adaptation would be nonsensical as "a computer [and thus an algorithm/platform] is unaware of a user's emotions, and it never expresses emotions of its own" [21, p. 82]. Other examples include "I feel understood by my manager" (Item 3), and "I don't feel very good about the way my manager talks to me" (Item 13). The extensive re-wording required to 
adapt the WCQ to an algorithmically managed work context would jeopardize its psychometric properties.

Although it may be possible to drop various items from the WCQ that cannot be adapted to the context of algorithmic management, this may also threaten the instrument's psychometric properties. Specifically, "autonomy-support" is a multi-dimensional concept and thus a formative construct. As such, the managerial behaviours that compose an autonomy-supportive social context, as well as the proportion of items allocated to each behaviour, should be given equal weight and representation in the associated instrument [19]. Thus, dropping items would necessitate adding back items to sufficiently account for the managerial behaviours that compose an autonomy-supportive context.

Finally, although other climate instruments exist, they are conceptually further from the context of platform-work than the WCQ which was conceived expressly for organizational contexts. Specifically, four other well-established Climate Questionnaires exist, namely the: Health Care Climate Questionnaire; Learning Climate Questionnaire; Sport Climate Questionnaire; and Perceived Parental Autonomy Support Scale. Similar to the WCQ, these scales concern the degree to which target individuals perceive people in positions of authority (e.g., healthcare providers, teachers, coaches, or parents) to be autonomysupportive. While these scales do not provide a sufficient basis from which to adapt measures, their existence demonstrates the sensitivity of the instrument to the context. Thus, a new instrument suited to the context of algorithmic management is both timely and needed to advance our understanding of platform workers' experienced autonomy.

\section{Methodology}

To generate a validated instrument for PAAS, we adopt MacKenzie et al.'s [16] rigorous and well-cited scale development process. Conceived for use by IS and behavioral science researchers, Mackenzie et al.'s 10step process begins with construct conceptualization and culminates in the development of scale norms. While all ten steps are important, "practical limitations may prevent researchers from being able to follow all of the recommendations" [16, p. 329]. As such, Mackenzie et al. stress placing "more attention on the front end of the process [rather] than on cross-validating the scale and developing norms for it", steps 9 and 10 respectively (ibid.).

Accordingly, we chose not to undertake steps 9-10 as both require data collections with sample sizes that are unrealistic for our current study. Rather, we focused on developing an instrument to measure PAAS on ridehailing platforms (e.g., Uber) which are considered to be paradigmatic instances of algorithmic management [2]. While this decision may limit the generalizability of our instrument to ride-hailing platforms, it is theoreticallyjustified [2, 5]. Moreover, the decision is further supported based on the novelty of the phenomenon, as well as MacKenzie et al.'s recommendations [16].

\subsection{Construct Conceptualization}

Drawing on the conceptual and empirical literature, we formally define PAAS as the degree to which a platform worker perceives that an algorithmicallymanaged digital labour platform maximizes their sense of volition, freedom, and choice by empowering their decision-making and work-methods authority in respect to the execution of their primary work tasks. Furthermore, based on the SDT, job-design, and gigeconomy literature, we conceptualize PAAS as a second-order formative construct with four first-order reflective constructs, namely: (i) acknowledging target users' perspectives; (ii) providing rationale to target users; (iii) offering choices to target users; and (iv) minimizing pressure on target users. Sub-dimensions are defined in Table 1, along with selected sources.

\begin{tabular}{|c|c|}
\hline Sub-dimensions and Definitions & Sources \\
\hline $\begin{array}{l}\text { Acknowledging perspectives: The platform considers } \\
\text { target users' situations and viewpoints by taking their } \\
\text { internal frame of reference when processing information } \\
\text { and enacting decisions. }\end{array}$ & $\begin{array}{l}{[6,12,} \\
13,22 \\
23]\end{array}$ \\
\hline $\begin{array}{l}\text { Providing rationale: The platform offers meaningful } \\
\text { explanations to target users for platform-related requests } \\
\text { and/or decisions. }\end{array}$ & $\begin{array}{l}{[6,12,} \\
13,22 \\
23]\end{array}$ \\
\hline $\begin{array}{l}\text { Offering choices: The platform provides target users } \\
\text { with choices in regard to key aspects of their job; it } \\
\text { empowers decision-making and work-methods authority } \\
\text { in respect to the execution of their primary work tasks. }\end{array}$ & $\begin{array}{l}{[12,13,} \\
22,23]\end{array}$ \\
\hline $\begin{array}{l}\text { Minimizing pressure: The platform avoids practices } \\
\text { that are controlling, intrusive, and dominating to compel } \\
\text { a target user's behavior. }\end{array}$ & $\begin{array}{l}{[12,13,} \\
23]\end{array}$ \\
\hline
\end{tabular}

Table 1: PAAS Sub-dimensions

\subsection{Item Generation}

Following Mackenzie et al.'s recommendations, we relied on a variety of sources to generate our initial pool of items. Beginning with the notion that the operational choices embedded in a platform's architecture will implicitly shape the platform workers' autonomy $[6,11$, $20,15]$ we then returned to the SDT and job-design literature, as well as the recent literature and surveys of platform workers, to formulate items for the four subdimensions of our perceived algorithmic autonomysupport construct. Through a review of the literature and deduction from the construct's conceptual definition, 24 items were generated for the four sub-dimensions. 
When testing the psychometric properties of a measure, researchers should generate more items than would be required in the final scale [18]. Thus, to generate more items, we conducted cognitive interviews with representatives of the population, namely Uber drivers $[16,24]$ that had been working for Uber for at least one month. The goals of these interviews were to: (i) review the items generated based on the literature to ensure that they represent the construct (e.g., face validity); (ii) ensure that the items generated were free from wording concerns; and (iii) generate new items based on the interviewee's responses reflections.

A convenience sample of ten interviews with Uber drivers was conducted (Sex: 6 men, 4 women; Average age: 38; Work status: $70 \%$ part-time; Tenure: $50 \%$ working for 2-6 months; $50 \%$ working > 24 months). Interviews were conducted virtually and, on average, lasted 54 minutes. Each interview began with a series of semi-structured questions after which interviewees were presented with the preliminary list of items and asked to indicate their degree of agreement or disagreement with each statement using a 5-point Likert scale ( $1=$ strongly disagree, 5 = strongly agree). Using a think-aloud protocol, participants were asked to read and answer the items aloud with the directive to 'say whatever comes to mind while completing the survey'. Our interviews led us to refine, drop, and add new items to yield a total of 33 items (see Table 2).

\begin{tabular}{|l|c|c|c|c|c|}
\hline Dimension & Original & Amended & Dropped & Added & Revised \\
\hline Acknowledging perspectives & 5 & 4 & 0 & 4 & 9 \\
\hline Offering choices & 9 & 4 & 4 & 4 & 9 \\
\hline Providing rationale & 3 & 3 & 0 & 3 & 6 \\
\hline Minimizing pressure & 6 & 2 & 0 & 3 & 9 \\
\hline \hline Total items & $\mathbf{2 3}$ & $\mathbf{1 3}$ & $\mathbf{4}$ & $\mathbf{1 4}$ & $\mathbf{3 3}$ \\
\hline
\end{tabular}

Table 2: Item Generation

\subsection{Content Validation}

Content validity concerns the systematic examination of an instrument's test items to ascertain whether each individual item proposed is representative of a dimension of the content domain of the construct, and whether collectively, all of the items are representative of the complete content domain of the first and second order constructs [16]. Given that a lack of content validity is a major problem for constructs with formative indicators, we conducted a two round, online Q-sort, as recommended by Petter et al. [17].

5.3.1. Q-sort participants. The initial round of our Qsort was conducted with five academic subject matter experts (SME) that were purposively sampled based on their knowledge of SDT, autonomy-support, and related scale development $[24,25]$. None of the contributing authors participated in the Q-sort. The second round of our Q-sort was conduced with Uber drivers. We chose to conduct our Q-sort with two different populations for a more robust analysis. Specifically, whereas academic subject matter experts can identify the behaviours that likely compose the criterion space of our construct, the exact boundaries of our construct within the context of Uber's algorithmically managed platform are best identified by the target population as they are the ones working with and experiencing the app [24, 26, 25].

5.3.2. Round 1. Our first round was conducted with five (5) SMEs. Each expert was presented with the definition of our PAAS construct and its four sub-dimensions, as well as a randomly sorted list of items. The experts were asked to sort the 33 items; after sorting the experts were given the opportunity to explain their rationale. For Round 1, the inter-judge raw agreement scores averaged 0.79 and Cohen's Kappa averaged 0.76. Following established guidelines for interpreting the Kappa coefficient [27], the value of 0.76 indicates a substantial level of agreement beyond chance for the SMEs in the first round. Per Table 3, the initial overall placement ratio of items within the target constructs was $80 \%$; this statistic is "a measurement of the overall frequency with which judges placed items within the intended theoretical construct, [and] is indicative of the reliability of the classification scheme" [26, p. 118].

\begin{tabular}{|l|c|c|c|c|c|c|}
\hline Target Category & AP & OC & PR & MP & Total & Hit \% \\
\hline Acknowledging Perspectives (AP) & $\mathbf{3 1}$ & 8 & 2 & 4 & 45 & $69 \%$ \\
\hline Offering Choices (OC) & 1 & $\mathbf{4 0}$ & 3 & 1 & 45 & $89 \%$ \\
\hline Providing Rationale (PR) & & & $\mathbf{2 9}$ & 1 & 30 & $97 \%$ \\
\hline Minimizing Pressure (MP) & & 8 & 5 & $\mathbf{3 2}$ & 45 & $71 \%$ \\
\hline Total placements: 165 & & \multicolumn{7}{|c|}{ Overall hit ratio: $80 \%$} \\
\hline
\end{tabular}

Table 3: Placement Ratios Summary (Round 1)

A review of the placement ratios (Table 3) indicates that while the 'Offering Choices' and 'Providing Rational' constructs both demonstrate a high degree of content validity, the 'Acknowledging Perspectives' and 'Minimizing Pressure' constructs demonstrate a lower degree of content validity. Following an examination of the off-diagonal items in Table 3 we found that the Acknowledging Perspectives item "The platform allows me to specify my work preferences (preferred locations, shifts, riders, etc.) using filters and other advanced settings" was placed in the 'Offering Choices' category by all five SMEs. While we agree with the experts' justifications that the item could be considered as 'Offering Choices', both our literature review and the results from our item-generation interviews $(n=10)$ strongly support that our population of interest considers this item as acknowledging their perspective.

Specifically, during the semi-structured portion of the interviews, we asked all respondents the following 
two questions: (1) What aspects of the Uber app (e.g., interface and/or algorithm) impede you from acting autonomously? And (2) What changes could be made to the Uber app that would help you to feel (and act) more autonomous(ly)? The item in question was conceived based on responses from all ten drivers indicating that they wish that the platform would allow them to set their own preferences with regards to drivers, shifts, and routes, in advance such that the automatic work allocation decisions made by the platform would consider their "situation" and "views" when assigning work. As such, the item was left under the 'Acknowledging Perspectives' dimension.

Importantly, in analyzing the 'Minimizing Pressure' items, we found that five of the nine items were placed in the 'Offering Choices' dimension by at least one expert. A review of the experts' justifications indicated that the likely cause of this issue was that the items were in fact measuring a presence of pressure (and thus, a lack of choice), rather than the minimization of pressure. Where eight out of the nine items were reversed coded, it became clear that our items were not measuring the correct dimension. Based on the experts' feedback, and guidelines in the literature cautioning against the use of reverse-coded items (e.g., [28]), we removed the reverse coding of the items, and revised our construct from 'Minimizing Pressure' to 'Presence of Pressure', defined as: The platform applies practices that are controlling, intrusive, and dominating to compel a target user's behavior. This decision is aligned with views of autonomy-support and psychological control as interpersonal styles that lie on opposite ends of the same theoretical continuum in terms of their psychological significance for people's autonomy [19].

Lastly, we noted that the 'Minimizing Pressure' item "The platform provides me with constructive feedback to improve my performance" was consistently placed in the 'Providing Rational' category. Based on the experts' justifications, we moved the item to the 'Providing Rationale' dimension for round two of our Q-sort.

5.3.3. Round 2. The second round of our Q-sort was conducted with two members of the target population. Given their unique experiences, Uber drivers were considered to be a good choice to reliably distinguish between aspects of the content domain as they pertain to the realities of the platform $[16,26]$.

\begin{tabular}{|l|c|c|c|c|c|c|}
\hline Target Category & AP & OC & PR & PP & Total & Hit \% \\
\hline Acknowledging Perspectives (AP) & $\mathbf{1 4}$ & 2 & 1 & 1 & 18 & $78 \%$ \\
\hline Offering Choices (OC) & 1 & $\mathbf{1 6}$ & 1 & & 18 & $89 \%$ \\
\hline Providing Rationale (PR) & & & $\mathbf{1 3}$ & 1 & 14 & $93 \%$ \\
\hline Presence of Pressure (PP) & & & & $\mathbf{1 6}$ & 16 & $100 \%$ \\
\hline \hline Total placements: 66 Total hits: 59 & \\
\hline
\end{tabular}

Table 4: Placement Ratios Summary (Round 2)
All constructs revealed high content validity; and our revised pressure construct reached a perfect $(100 \%)$ hit ratio (see Table 4). Moreover, the 'Acknowledging Perspectives' item that was consistently misclassified as 'Offering Choices' by the SMEs, was correctly classified by the drivers.

In view of the results from both rounds of our Q-sort, a decision was made not to drop any items at this stage, given that the few items considered for dropping (based on the inter-rater reliability, or IRR, and potential for fitting in more than one category in Round 1) were deemed theoretically important to algorithmicallymanaged platform work, as confirmed by drivers in Round 2 of our Q-sort. Rather, with the goal of retaining four to six items per construct, we will rely on the use of exploratory factor analysis (EFA) to uncover the underlying structure and to drop items that cross-load or have low factor loadings [18]. Items resulting from our content validation are listed in the Appendix (Table I).

\subsection{Measurement Model Specification}

Prior to statistically validating our construct, it is necessary to formally specify the measurement model. As previously elaborated, PAAS is conceptualized as a multidimensional, and thus formative construct, based on the SDT literature and its application in the organizational context wherein the notion of autonomy support in organizations has been conceptualized as a cluster of supervisory behaviors that are theorized to support autonomy [12]. Specifically, perceived algorithmic autonomy support is formed by the four proposed dimensions (behaviors) where there is independence between the dimensions; and each dimension contributes independently to the totality and the configuration of the perceptions of the platform's algorithmic autonomy support $[16,17]$. As a result of our measurement model's structure, we will use validation techniques suitable for reflective measures only at the first-order level and different validation techniques for our second-order formative construct.

\subsection{Survey study}

Following content validation, our next steps are to: (i) examine the psychometric properties of our new scale; and (ii) assess the scale's validity. To do so, an online survey has been created which includes our preliminary PAAS instrument (33 items), two additional scales required to test nomological validity, and a set of demographic and control questions. We now elaborate our progress in this survey study and our next steps.

5.5.1. Pilot test. A pilot test has been conducted with a total of 25 respondents which included 23 Uber drivers 
and two (2) PhD candidates, one of which works as a part-time Uber driver. Pilot test participants were recruited through Uber driver forums and social media groups, personal contacts, and referrals. The pilot test allowed us to establish the time required to complete the survey (to establish appropriate incentives for our main survey), as well as to gain feedback on the content and user experience. Various participant instructions and question formats were revised through the pilot survey.

5.5.2. Scale purification and refinement. Following our pilot test, a pre-test of our survey is required for the purposes of exploratory factor analyses (EFA). Our pretest survey has been distributed via Prolific, a recently established crowdworking platform similar to Amazon Mechanical Turk (AMT). Unlike AMT however, the Prolific platform functions strictly as an online subject recruitment which explicitly caters to researchers. Recent studies have supported Prolific to be a more ethical platform to recruit participants, as well as one that offers a more diverse population and better quality data than MTurk [29]. Our survey is being administered via Qualtrics, an online survey platform.

Our survey is currently being promoted on Prolific with a target sample size of 200 Uber drivers in North America. This sample size is considered sufficient for the purposes of EFA as it exceeds both the "10 times" rule and the minimum item-to-response ratio of 5:1 [16, 30]. To qualify for participation, respondents had to be at least 18 years old and have completed at least one month of work on the Uber platform to ensure sufficient familiarity and experience with the platform, its functions, and features.

Once our data has been collected and cleaned, EFA will be conducted using principal components analysis (PCA) on SPSS [26]. Best practices [e.g., 13, 14, 15] and established thresholds will be applied for removing items and for evaluating the underlying structure of our multi-dimensional PAAS construct. Convergent validity will be further assessed by calculating the average variance extracted (AVE) for each first-order reflective dimension and discriminant validity will be assessed using the Fornell-Larcker criterion [26].

5.5.3 Scale and Nomological Validation. The final steps in our process will include scale validation and nomological net testing using a new data sample. Nomological validity refers to the degree to which a specific measure makes accurate predictions of other concepts in a theoretically based model. Based on selfdetermination theory and organizational support theory, PAAS should positively impact job satisfaction (H1) and perceived organizational support $(\mathrm{H} 2)[3,12,13]$.

Following Mackenzie et al.'s [16] suggestion of combining steps to reduce costs, scale validity will be assessed concomitantly with its nomological validity. To do so, we will run the same study as in our pre-test but with a new sample of 250 North American Uber drivers. As recommended by Gefen et al. [30], Daniel Soper's online calculator [31] was used to calculate the minimum sample size needed to achieve adequate power, and to detect our anticipated effect sizes given the structural complexity of the model.

Existing scales will be adopted to measure job satisfaction and POS. For job satisfaction, we follow Eisenberger et al.'s [32] use of four items from Quinn and Shepard's Overall Job Satisfaction index $(\alpha=0.79)$, a facet-free job satisfaction scale measured on a 7-point Likert-scale ( 1 = strongly disagree, 7 = strongly agree $)$. Sample items are "All in all, I am very satisfied with my current job" and "Knowing what I know now, if I had to decide all over again whether to take my job, I would".

To measure POS, we will use Eisenberger et al.'s [33] short version of the Survey of Perceived Organizational Support $(\alpha=0.97)$ which consists of eight items measuring POS on a 7-point Likert-scale ( 1 = strongly disagree, 7 = strongly agree). Sample items are "The organization really cares about my wellbeing." and "The organization fails to appreciate any extra effort from me. $(R)$ ". Basic demographics, including our control variables (tenure, work status, and algorithmic knowledge) will also be collected.

Confirmatory factor analysis (CFA) of the measurement model will be conducted to confirm the psychometric properties of our scales. SmartPLS will be used to assess the internal consistency, convergent, and discriminant validity of our model's constructs. The two main effects in our study $(\mathrm{H} 1-\mathrm{H} 2)$ will be tested using the PLS-SEM approach. This approach is adopted given the presence of a formative variable (PAAS) in our nomological model, and given that the identification of relationships is a central purpose in our research [30].

\section{Expected Contributions}

In recent years, advances in algorithm technologies have enabled the creation of sociotechnical systems which "interweave users and their social structures with technologies" more closely than ever before [34, p. 37]. As algorithmic systems have expanded into various social sectors, their application to the management of workers has become a key concern for scholars studying the gig-economy and the digital transformation of work [8]. Despite growing interests, our understanding of the impacts of sociotechnical phenomena such as digital labour platforms and other AI-driven artefacts on human users is still nascent [2, 3, 5, 9]. For instance, whereas earlier approaches conceived of algorithms as either augmenting or reducing human agency, recent work 
tells us that the interaction between human and machine agents is complex and needs more differentiation [8].

Our main contribution in this paper is the generation of a validated instrument which can be used to measure perceptions of algorithmic autonomy-support. To the best of our knowledge, this paper is the first to generate and validate an instrument to measure perceived algorithmic autonomy-support. Given competing narratives on workers' experiences of agency and autonomy, our instrument sets the stage for scholars to empirically measure workers' perceptions of algorithmic autonomy-support across different contexts; this should allow scholars to gain a more nuanced, and empirical, understanding of the sociotechnical factors contributing to such perceptions.

Moreover, in validating our construct in its nomological net, we also aim to shed light on how perceptions of algorithmic autonomy-support can affect job satisfaction and perceived organizational support. These contributions are important, given that many scholars have suggested that algorithmic management and the "platformization" of work have contributed to a lack of job satisfaction and diminished feelings of organization support in the gig-economy $[4,11]$.

With the emerging importance of the algorithm, a key question is how to make better human-centered algorithms that are useful, fair, and accepted by the various parties affected by them [34]. Yet as sociotechnical ecosystems powered by algorithms [4, 34], digital labour platforms have continued to raise concerns among the public and policy makers about the implications of algorithmic management and workplace surveillance on workers' agency and autonomy, their well-being, motivation, and performance $[4,6,7,10]$.

In the unique context of the gig-economy, where managerial activities are outsourced to system designers that develop and manage algorithms, achieving a better understanding of how digital labour platforms and algorithms should be designed has been outlined as a key area of research [9]. Given the well-recognized benefits of promoting workers' autonomy, we consider that the key to ethically unlocking the potential of digital labour platforms lies in promoting workers' autonomy. By focusing on the interrelatedness of both the human and technical aspects of a platform organization to reconceptualise SDT's notion of autonomy-support, we hope to provide scholars and practitioners with a framework with which to evaluate and guide the design of digital labour platforms that successfully optimize both workers' well-being and technical performance.

\section{Next Steps and Future Considerations}

With our survey study underway, we expect to complete our data collection and analyses within the coming weeks; we look forward to publishing our results. Notably, we are also running a concomitant study in which we explore the impacts of perceptions of algorithmic fairness on workers' perceptions of organizational support, as well as the relationship between PAAS and perceived algorithmic fairness.

Given the pace at which new platforms emerge, and considering that algorithmic management is moving beyond the realm of the gig-economy to become adopted in traditional organizations, the ability to empirically study perceptions of algorithmic autonomysupport, and their impact on other variables, is of great importance $[3,6,11]$. We hope that our finalized instrument will be used to further such endeavours.

To best support such research, we acknowledge that future work will be required to generalize our instrument. Specifically, given that we developed and tested our instrument solely for ride-hailing platforms, in this case Uber, we recognize that various items in our scale may be less applicable to other labour platforms. Although words such as "riders" can be replaced with "clients", and "rides" can be replaced by "jobs" (e.g., UpWork) or "tasks" (e.g., TaskRabbit) to suit the particular platform context, certain items may be entirely irrelevant in different contexts. Thus, a future research opportunity that we intend to undertake is the cross-validation of our instrument in other algorithmically-managed platform work contexts. Given the number of labour platforms in existence and the rate at which they continue to emerge, we hope that other scholars will join us in this pursuit by exploring PAAS on digital labour platforms with varying levels of algorithmic control (e.g., centralized vs. decentralized), as well as those that mediate different types of labour (e.g., virtual vs. physical), or that require differing skilllevels (e.g., high vs. low).

\section{Acknowledgements}

We are grateful for the constructive comments of the anonymous reviewers, which are being used to further prepare this work for a journal submission. This research is supported by Insight Grant 201974 (20182022) from the Social Sciences and Humanities Research Council of Canada (SSHRC).

\section{References}

[1] A. Quinn and B. Bederson, "Human Computation: A Survey and Taxonomy of a Growing Field," in CHI 2011 - Session: Crowdsourcing, Vancouver, 2011.

[2] M. Möhlmann and L. Zalmanson, "Hands on the wheel: Navigating algorithmic management and Uber 
drivers'," in International Conference on Information Systems, Seoul, South Korea, 2017.

[3] N. Jabagi, A.-M. Croteau and L. K. Audebrand, "Perceived Organizational Support in the Face of Algorithmic Management: A Conceptual Model," in Proceedings of the 53rd HICSS, Maui, Hawaii, 2020.

[4] Ajunwa, I. and G. D., "Platforms at Work: Automated Hiring Platforms and Other New Intermediaries in the Organization of Work.," Work and Labor in the Digital Age, pp. 61-91, 2019.

[5] M. K. Lee, D. Kusbit, E. Metsky and L. Dabbish, "Working with Machines: The Impact of Algorithmic, Data-Driven Management on Human Workers," in 33rd Annual ACM SIGCHI Conference, 2015.

[6] A. Shapiro, "Between autonomy and control: Strategies of arbitrage in the "on-demand" economy," New Media \& Society, vol. 20, no. 8, pp. 2954-2971, 2018.

[7] M. H. Jarrahi and W. Sutherland, "Algorithmic Management and Algorithmic Competencies: Understanding and Appropriating Algorithms in Gig work," in iConference, Washington, 2018.

[8] C. Katzenbach and L. Ulbricht, "Algorithmic governance," Internet Policy Review, vol. 8, no. 4, pp. $1-18,2019$.

[9] M. de Reuver, C. Sørensen and R. Basole, "The Digital Platform: A Research Agenda," Journal of Information Technology, vol. 33(2), pp. 124-135, 2018.

[10] X. N. Deng and K. Joshi, "Is Crowdsourcing a Source of Worker Empowerment or Exploitation? Understanding Crowd Workers' Perceptions of Crowdsourcing Career," in 34th ICIS, Milan, 2013.

[11] K. Kuhn and A. Maleki, "Micro-entrepreneurs, dependent contractors, and instaserfs: understanding online labor platform workforces," The Academy of Management Perspectives, vol. 31, no. 3, pp. 183-200, 2017.

[12] E. L. Deci, A. H. Olafsen and R. M. Ryan, "SelfDetermination Theory in Work Organizations: The State of a Science," The Annual Review of Organizational Psychology and Organizational Behavior, vol. 4, p. 19-43, 2017.

[13] P. Baard, E. Deci and R. Ryan, "Intrinsic need satisfaction: a motivational basis of performance and well-being in two work settings," Journal of Applied Social Psychology, vol. 34, no. 10, pp. 2045-68, 2004.

[14] W. Orlikowski and S. Scott, "The Algorithm and the Crowd: Considering the Materiality of Service Innovation," MIS Quarterly, vol. 39, no. 1, pp. 201216, 2015.

[15] N. Jabagi, A.-M. Croteau, L. K. Audebrand and J. Marsan, "Gig-workers' motivation: thinking beyond carrots and sticks," Journal of Managerial Psychology, vol. 34, no. 4, pp. 192-213, 2019.

[16] S. B. MacKenzie, P. M. Podsakoff and N. P. Podsakoff, "Construct Measurement and Validation Procedures in MIS and Behavioral Research:
Integrating New and Existing Techniques," MIS Quarterly, vol. 35, no. 2, pp. 293-334, 2011.

[17] S. Petter, D. Straub and A. Rai, "Specifying Formative Constructs in Information Systems Research," MIS Quarterly, vol. 31, no. 4, p. 623-656, 2007.

[18] T. Hinkin, "A Brief Tutorial on the Development of Measures for Use in Survey Questionnaires," Cornell University, School of Hotel Administration, 1998.

[19] G. A. Mageau, F. Ranger, M. Joussemet, R. Koestner, E. Moreau and J. Forest, "Validation of the Perceived Parental Autonomy Support Scale (P-PASS)," Canadian Journal of Behavioural Science, vol. 47, no. 3, p. 251-262, 2015.

[20] W. Sutherland and M. Jarrahi, "The Sharing Economy and Digital Platforms: A Review and Research Agenda," International Journal of Information Management, vol. 43, pp. 328-341, 2018.

[21] C. Nass and Y. Moon, "Machines and Mindlessness: Social Responses to Computers," Journal of Social Issues, vol. 56, no. 1, pp. 81-2000, 2000.

[22] M. Gagné, R. Koestner and M. Zuckerman, "Facilitating acceptance of organizational change: the importance of self-determination," J. Appl. Soc. Psychol, vol. 30, no. 9, pp. 1843-52, 2000.

[23] G. R. Slemp, M. L. Kern, K. J. Patrick and R. M. Ryan, "Leader autonomy support in the workplace: A meta-analytic review," Motivation and Emotion, vol. 42, no. 5, p. 706-724, 2018.

[24] M. Howard, "Scale Pretesting," Practical Assessment, Research \& Evaluation, vol. 23, no. 5, pp. 1-14, 2018

[25] G. O. Boateng, T. B. Neilands, E. A. Frongillo, H. R. Melgar-Quiñonez and S. L. Young, "Best Practices for Developing and Validating Scales for Health, Social, and Behavioral Research: A Primer.," Frontiers in public health, vol. 6, no. 149, 2018.

[26] S. Moussawi and M. Koufaris, "Perceived Intelligence and Perceived Anthropomorphism of Personal Intelligent Agents: Scale Development and Validation," in Proceedings of the 52nd Hawaii International Conference on System Sciences, Wailea, Hawaii, 2019.

[27] J. Landis and C. Koch, "The Measurement of Observer Agreement for Categorical Data," Biometrics, vol. 33, no. 1, pp. 159-74, 1977.

[28] P. Podsakoff, S. MacKenzie, J. Lee and N. Podsakoff, "Common Method Biases in Behavioral Research: A Critical Review of the Literature and Recommended Remedies," Journal of Applied Psychology, vol. 88, no. 5, p. 879-903, 2003.

[29] S. Palan and C. Schitter, "Prolific.ac-A subject pool for online experiments," Journal of Behavioral and Experimental Finance, vol. 17, no. March, pp. 22-27, 2018.

[30] D. Gefen, E. Rigdon and D. Straub, "An Update and Extension to SEM Guidelines for Administrative and Social Science Research.," MIS Quarterly, vol. 35, no. 2, p. iii-xiv, 2011. 
[31] D. Soper, "Calculator: A-priori Sample Size Calculator for Structural Equation Models," n.d.. [Online]. Available: www.danielsoper.com/statcalc/ . [Accessed 1210 2019].

[32] R. Eisenberger, J. Cummings, S. Armeli and P. Lynch, "Perceived organizational support, discretionary treatment, and job satisfaction," Journal of Applied Psychology, vol. 82, pp. 812-820, 1997.
[33] R. Eisenberger, R. Huntington, S. Hutchison and D. Sowa, "Perceived organizational support," Journal of Applied Psychology, vol. 71, pp. 500-507, 1986.

[34] D. Shin, "Socio-Technical Design of Algorithms: Fairness, Accountability, and Transparency," in 30th European Conference of the International Telecommunications Society (ITS): "Towards a Connected and Automated Society", Helsinki, 2019.

\section{Appendix - Table I}

\begin{tabular}{|c|c|c|}
\hline \multirow{9}{*}{ 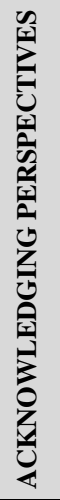 } & 1 & The platform gathers relevant information to understand my personalized needs. \\
\hline & 2 & $\begin{array}{l}\text { The platform allows me to specify my work preferences (preferred locations, shifts, riders, etc.) using filters and } \\
\text { other advanced settings. }\end{array}$ \\
\hline & 3 & $\begin{array}{l}\text { The platform gathers relevant personal performance data before suggesting a new way to do things via app } \\
\text { notifications or email. }\end{array}$ \\
\hline & 4 & The platform allows me to accurately rate clients. \\
\hline & 5 & $\begin{array}{l}\text { When a client rates me, the platform does not include unfair or abusive clients' feedback in my overall } \\
\text { performance (star) rating. }\end{array}$ \\
\hline & 6 & The platform provides me with a way to effectively contest unfair or abusive clients' feedback. \\
\hline & 7 & The platform sets realistic expectations for me based on my personal situation and performance. \\
\hline & 8 & When I contact customer service through the app, I feel that my thoughts and feelings are understood. \\
\hline & 9 & When I contact customer service through the app, I feel that my opinion and point of view are considered. \\
\hline \multirow{9}{*}{ 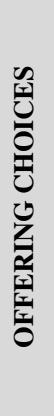 } & 10 & The platform allows me to choose more desirable rides (clients). \\
\hline & 11 & The platform allows me to control my compensation per ride. \\
\hline & 12 & I am free to choose the way I carry out my work. \\
\hline & 13 & I have control over the scheduling of my work. \\
\hline & 14 & I have control over what I am supposed to accomplish when working on the platform. \\
\hline & 15 & I set my own goals while working on the platform. \\
\hline & 16 & The platform provides me with the information I need to take adequate decisions concerning my work. \\
\hline & 17 & The platform's tools and features enable me take adequate decisions concerning my work. \\
\hline & 18 & The platform provides sufficient tools and features for me to do my job the way I want. \\
\hline \multirow{7}{*}{ 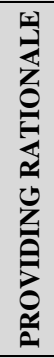 } & 19 & $\begin{array}{l}\text { When the platform sends me performance advice (via app notifications or email), it explained why they wanted me } \\
\text { to do it. }\end{array}$ \\
\hline & 20 & The platform provides information about how it matches riders and clients. \\
\hline & 21 & When the platform does not allow me to do something, it explains why. \\
\hline & 22 & When the platform offers me a reward or promotion, it explains why. \\
\hline & 23 & I understand why the platform has not offered me a reward or promotion yet. \\
\hline & 24 & Platform penalties (e.g., deactivations, lost fares and promotions) are clearly explained. \\
\hline & 25 & The platform provides me with constructive feedback to improve my performance. \\
\hline \multirow{8}{*}{ 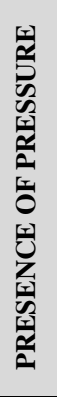 } & 26 & When the platform provides feedback on my performance, I must follow their advice. \\
\hline & 27 & I feel penalized by the platform when declining a ride. \\
\hline & 28 & I feel penalized by the platform when cancelling a ride. \\
\hline & 29 & I feel obligated to accept all rides. \\
\hline & 30 & When conducting my work, I feel constantly monitored by the platform. \\
\hline & 31 & $\begin{array}{l}\text { The platform rating system (Stars, Acceptance, and Cancellation rates) prevents me from doing my job the way I } \\
\text { want. }\end{array}$ \\
\hline & 32 & When working on the platform, I often feel like I have to follow the platform's commands. \\
\hline & 33 & When working on the platform, I feel forced to do things I do not want to do. \\
\hline
\end{tabular}

